\title{
Dimensional Reduction in Quantum Dipolar Antiferromagnets
}

\author{
P. Babkevich, ${ }^{1,}{ }^{*}$ M. Jeong, ${ }^{1}$ Y. Matsumoto, ${ }^{2}$ I. Kovacevic, ${ }^{1}$ A. Finco, ${ }^{1,3}$ R. Toft-Petersen, ${ }^{4}$ \\ C. Ritter, ${ }^{5}$ M. Månsson, ${ }^{1,6,7}$ S. Nakatsuji, ${ }^{2}$ and H. M. Rønnow ${ }^{1}$ \\ ${ }^{1}$ Laboratory for Quantum Magnetism, Institute of Condensed Matter Physics (ICMP), \\ Ecole Polytechnique Féderale de Lausanne (EPFL), CH-1015 Lausanne, Switzerland \\ ${ }^{2}$ Institute for Solid State Physics, University of Tokyo, Kashiwa, Chiba 277-8581, Japan \\ ${ }^{3}$ ICFP, Département de physique, École normale supérieure, 45 rue d'Ulm, 75005 Paris, France \\ ${ }^{4}$ Helmholtz-Zentrum Berlin für Materialien und Energie, D-14109 Berlin, Germany \\ ${ }_{5}^{5}$ Institut Laue-Langevin, BP 156, F-38042, Grenoble Cedex 9, France \\ ${ }^{6}$ Laboratory for Neutron Scattering, Paul Scherrer Institut, CH-5232 Villigen, Switzerland \\ ${ }^{7}$ Department of Materials and Nanophysics, KTH Royal Institute of Technology, SE-164 40 Kista, Sweden \\ (Received 13 January 2016; revised manuscript received 16 March 2016; published 10 May 2016)
}

We report ac susceptibility, specific heat, and neutron scattering measurements on a dipolar-coupled antiferromagnet $\mathrm{LiYbF}_{4}$. For the thermal transition, the order-parameter critical exponent is found to be 0.20 (1) and the specific-heat critical exponent $-0.25(1)$. The exponents agree with the $2 \mathrm{D} X Y / h_{4}$ universality class despite the lack of apparent two-dimensionality in the structure. The order-parameter exponent for the quantum phase transitions is found to be $0.35(1)$ corresponding to $(2+1) \mathrm{D}$. These results are in line with those found for $\mathrm{LiErF}_{4}$ which has the same crystal structure, but largely different $T_{N}$, crystal field environment and hyperfine interactions. Our results therefore experimentally establish that the dimensional reduction is universal to quantum dipolar antiferromagnets on a distorted diamond lattice.

DOI: 10.1103/PhysRevLett.116.197202

Critical phenomena near continuous phase transitions do not depend on the microscopic details of systems but only on the symmetry of the order parameter and interactions and the spatial dimensionality [1]. Such universality for classical thermal transitions has been thoroughly demonstrated with various physical systems over decades while nowadays a similar line of effort is actively pursued for zero-temperature quantum transitions [2-4]. Comparing experimental observations with theoretical models has been particularly successful for magnetic insulators that could be simply modeled by short-ranged, exchange-coupled spins on a lattice. Although dipolar interactions appear to be more classical than their exchange-coupled counterparts, it has been shown that on a square or diamond lattice, quantum fluctuations can map long-ranged dipolar interactions to a two-dimensional Ising model [5-7]. The $\mathrm{Li} \mathrm{FF}_{4}$ family is special as the rare-earth ions are arranged in a slightly distorted diamondlike structure making them intriguing to study in relation to order by disorder phenomena [8].

For the case of a dipolar-coupled Ising ferromagnet, the theoretical upper critical dimension $\mathrm{D}^{*}=3$ and the meanfield calculations actually apply quite well as shown, for instance, in $\mathrm{LiHoF}_{4}$ [9]. This is despite the significant role of hyperfine interactions around the quantum phase transition [10,11]. Recently, quantum and classical critical properties of a long-range, dipolar-coupled antiferromagnet could be investigated for the first time with $\mathrm{LiErF}_{4}$ [12]. It was discovered that the specific-heat and order-parameter critical exponents, $\alpha=-0.28(4)$ and $\beta_{T}=0.15(2)$, for the thermal transition are totally different from the mean-field predictions of $\alpha=0$ and $\beta_{T}=0.5$. Instead, these exponent values suggest a $2 \mathrm{D} X Y / h_{4}$ universality class, despite the absence of any apparent two dimensionality in the structure of the system. This intriguing dimensional reduction was further corroborated by the $\beta_{H}=0.31(2)$ for the quantum transition induced by applying a longitudinal magnetic field, which corresponds to $(2+1) \mathrm{D}$, as expected from quantum-classical mapping [4]. Whether the dimensional reduction is universal to all dipolar quantum antiferromagnets or is special to $\mathrm{LiErF}_{4}$, due to rather close $(3 \mathrm{meV})$ higher-lying crystal-field levels or weak hyperfine interactions, is to date unknown.

Among the $\mathrm{Li} \mathrm{FF}_{4}$ family, where $R$ is a rare-earth ion, $\mathrm{LiYbF}_{4}$ has been suggested to be an alternate candidate for a dipolar antiferromagnet [13]. However, there are marked differences between $\mathrm{LiYbF}_{4}$ and $\mathrm{LiErF}_{4}$. First, the electronic level scheme is quite different with crystalline electric field split first excited state an order of magnitude higher in $\mathrm{LiYbF}_{4}$. Second, in $\mathrm{Yb}^{3+}$, there are two stable isotopes of $\mathrm{Yb}$ with strong hyperfine coupling $-11.0 \mu \mathrm{eV}$ for ${ }^{171} \mathrm{Yb}(14.3 \%)$ and $-3.0 \mu \mathrm{eV}$ for ${ }^{173} \mathrm{Yb}(16.1 \%)$. $\mathrm{LiErF}_{4}$ contains ${ }^{167} \mathrm{Er}(22.8 \%)$ whose hyperfine coupling strength is weak, $0.5 \mu \mathrm{eV}$. Therefore, $\mathrm{LiYbF}_{4}$ could serve as an excellent candidate to test for the robustness of dimensional reduction in dipolar antiferromagnets arranged on a distorted diamond lattice.

In this Letter, we present ac susceptibility, specific heat, and neutron scattering measurements on $\mathrm{LiYbF}_{4}$ and 

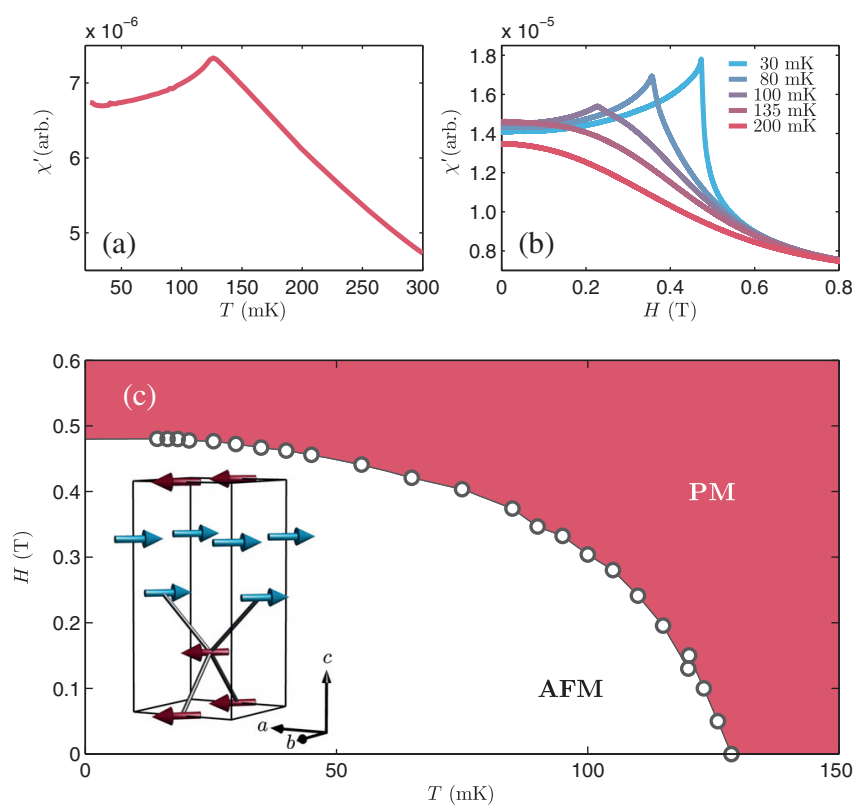

FIG. 1. (a) Real part of ac susceptibility $\chi^{\prime}$ as a function of temperature in zero field and (b) $\chi^{\prime}$ as a function of field at different temperatures. (c) Magnetic phase diagram mapped out using the susceptibility. Inset shows the bilayer magnetic structure of $\mathrm{LiYbF}_{4}$.

demonstrate the thermal and quantum critical properties. The field-temperature $(H-T)$ phase diagram is first mapped out and a bilayered $X Y$ antiferromagnetic order for the ground state is identified. Then we show that the critical exponents $\alpha, \beta_{T}$, and $\beta_{H}$ support the dimensional reduction as a universal feature of quantum dipolar antiferromagnets.

Large, high-quality single crystals were obtained from a commercial source. In order to reduce neutron absorption, the samples were enriched with the ${ }^{7} \mathrm{Li}$ isotope. The ac susceptibility $\chi(T, H)$ was measured on a single crystal using a mutual inductance method where the excitation field was $40 \mathrm{mOe}$ and the excitation frequency $545 \mathrm{~Hz}$. The specific heat $C_{p}(T)$ was measured by the relaxation method in a dilution refrigerator with a temperature stability of $0.1 \mathrm{mK}$. Powder neutron diffraction was performed using the high-intensity D1B and high-resolution D2B diffractometers at ILL, France, using incident neutron wavelength 2.52 and $1.59 \AA$, respectively. The evolution of the magnetic Bragg peak intensities with temperature and field was followed by performing high-resolution single-crystal neutron scattering using the triple-axis spectrometer FLEXX at HZB, Germany [14]. The instrument was set up with $40^{\prime}$ collimation before and after the sample and incident neutron wavelength of $\lambda=4.05 \AA$. The corresponding wave vector and energy resolution (FWHM) was on the order of $0.014 \AA$ and $0.15 \mathrm{meV}$, respectively.

Figure 1 shows bulk ac susceptibility data from a singlecrystal $\mathrm{LiYbF}_{4}$. The temperature-field phase boundary was mapped for a transverse magnetic field applied along the $c$ axis. Figure 1(a) shows the real part of the ac susceptibility $\chi^{\prime}$ as a function of temperature in zero field. The peak in zero field reflects the antiferromagnetic transition at $T_{N}=130 \mathrm{mK}$. Figure 1(b) shows $\chi^{\prime}(H)$ at 30-200 mK. Below $T_{N}$, a pronounced cusp is observed which corresponds to a quantum transition from the ordered to a quantum paramagnetic phase. At base temperature, a maximum in $\chi^{\prime}(H)$ is found at $H_{c}=0.48 \mathrm{~T}$. The peak shifts to lower fields as temperature is increased. Based on these measurements, we can accurately map out the phase diagram shown in Fig. 1(c).

The specific heat as a function of temperature is shown in Fig. 2(a). In zero field, a sharp peak in the specific heat capacity marks the second-order thermal transition [15]. On applying a transverse field, we find the peak at $T_{N}$ decreases in amplitude and shifts to lower temperature at $H=0.45 \mathrm{~T}$. Above $H_{c}$, only a broad hump is found in the specific heat capacity. At such low temperatures, phonon
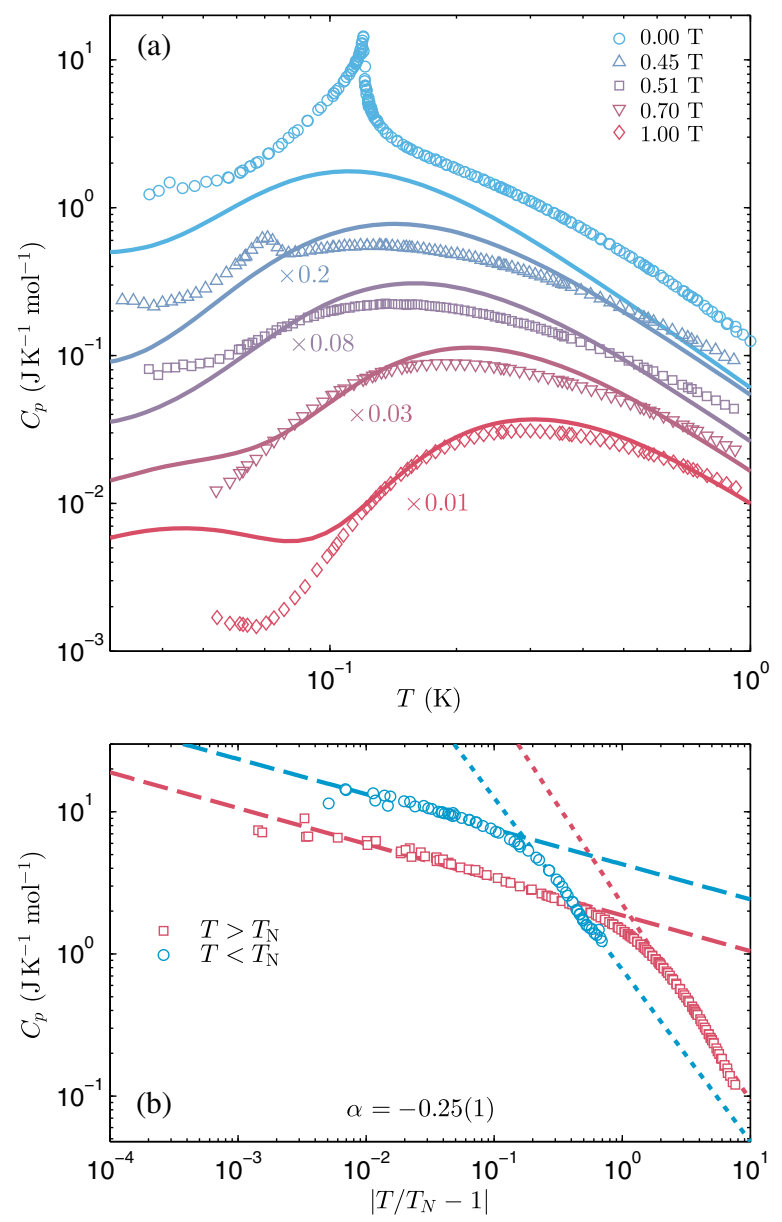

FIG. 2. (a) Specific heat in zero and finite fields as a function of temperature. Calculation of specific heat capacity in the singleion limit for different fields are plotted by continuous lines. The data were displaced vertically by multiplying with scaling factors given in the figure. (b) Determination of the specific-heat critical exponent $\alpha$ for the thermal transition based on measurements above and below $T_{N}$ (dashed line). Scaling away from the critical region was fitted by the dotted line. 
and crystal-field-level contributions are frozen out. We model the specific heat capacity away from the QPT using a parameter-free model where the Hamiltonian $\mathcal{H}$ contains crystal field, hyperfine, and Zeeman terms. From the diagonalized Hamiltonian $\langle n|\mathcal{H}| n\rangle=\epsilon_{n}$, we calculate for each isotope $i$ the Schottky specific heat, $C_{i}^{\text {Sch }}=k_{B} \beta^{2}\left[\left\langle\epsilon^{2}\right\rangle-\langle\epsilon\rangle^{2}\right]$, where $k_{B}$ is the Boltzmann factor and $\beta=1 /\left(k_{B} T\right)$. The thermal ensemble average is denoted by $\langle\ldots\rangle$. The total specific heat capacity is found from the weighted sum of contributions from each $\mathrm{Yb}$ isotope. The comparison between the experiment and our simple model is remarkably good considering that this is a parameter-free calculation with all parameters fixed from other experiments. It is possible to improve the comparison by including quadrupolar operators, and by fine-tuning hyperfine coupling strengths and the crystal field parameters, etc. However, this would give too many adjustable parameters, and the calculation anyway ignores collective effects beyond the mean-field level.

In zero applied field, close to $T_{N}$, the heat capacity can be described by a universal power law,

$$
C_{p}^{\mathrm{crit}}=A|t|^{\alpha}+B,
$$

where the reduced temperature $t=1-T / T_{N}, A$ and $B$ are free parameters which can have different values above and below $T_{N}$. The results of our analysis are shown in Fig. 2(b). The contribution from the background term, $B$, is found to be small and is set to zero above and below $T_{N}$. A good fit is found for $\alpha=-0.25(1)$, similar to the value of -0.28 (4) found in $\mathrm{LiErF}_{4}$ [12]. The negative exponents imply that $C_{p}$ is finite at $T_{N}$. Away from the phase transition we observe a change in the scaling. Above around $250 \mathrm{mK}$ and below $100 \mathrm{mK}$ the data can be fit to an exponent of around $-1.3(1)$. It is somewhat surprising that the critical scaling can be traced out all the way to $2 T_{N}$ and is dramatically different to $\mathrm{LiErF}_{4}$ where a crossover was found above $1.03 T_{N}$ [12].

To elucidate the magnetic structure below $T_{N}$, we performed neutron diffraction on a powder of $\mathrm{LiYbF}_{4}$. At $10 \mathrm{~K}$, in the paramagnetic phase, the crystal lattice was refined using the $I 4_{1} / a$ space group, where $a=5.13433(8) \AA$ and $c=10.5917(2) \AA$. Below $140 \mathrm{mK}$ we find additional peaks which emerge from antiferromagnetic ordering corresponding to a $\mathbf{k}=(1,0,0)$ magnetic propagation wave vector. Figure 3(a) shows a powder diffraction pattern obtained by subtracting measurements above $T_{N}$ from $50 \mathrm{mK}$ data. The magnetic peaks are well described by a bilayer antiferromagnetic structure with moments along the [110] direction, where moments related by $I$ centering are aligned antiparallel. An ordered moment of $1.9(1) \mu_{\mathrm{B}}$ is found to reside on each $\mathrm{Yb}^{3+}$ ion. A schematic of a possible magnetic structure is shown in Fig. 1(c). This differs from $\mathrm{LiErF}_{4}$ where the moments are parallel to the [100] direction. Although our data do not allow us to uniquely identify the magnetic structure,
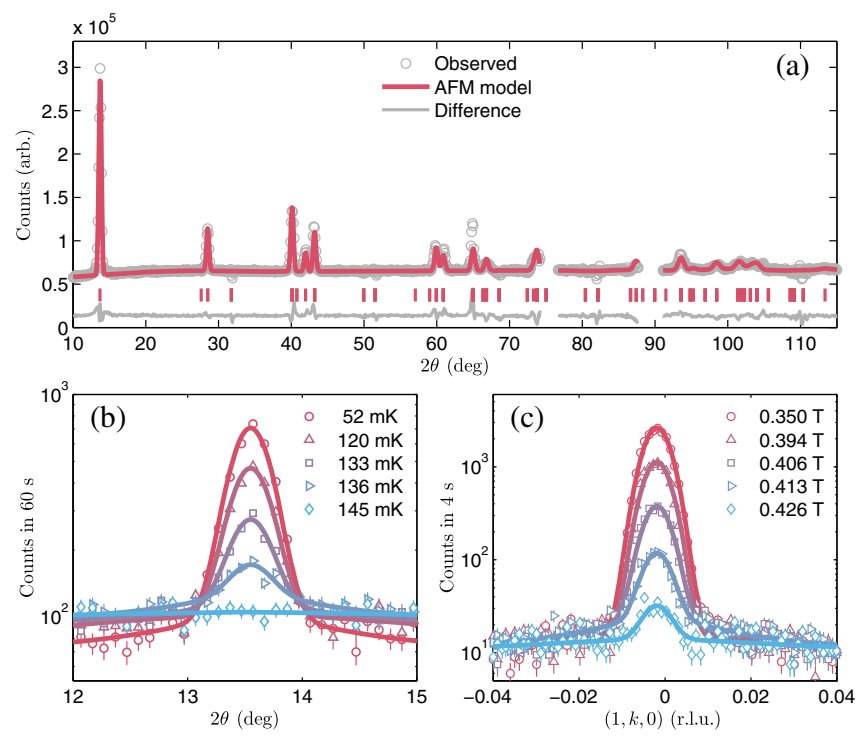

FIG. 3. (a) Magnetic powder diffraction pattern from the subtraction of paramagnetic background from $50 \mathrm{mK}$ measurements. (b) Magnetic Bragg peak from powder diffraction at different temperatures in zero field and (c) single-crystal measurements at $70 \mathrm{mK}$ in different fields. Lines are fits to a Gaussian with additional contribution from critical scattering.

it is clear that $\mathrm{LiErF}_{4}$ and $\mathrm{LiYbF}_{4}$ order differently (see Supplemental Material [16]). The origin of this is not entirely obvious, but could be attributed to the in-plane anisotropy set by the crystal field. This would depend primarily on the $B_{4}^{4}(c) \mathbf{O}_{4}^{4}(c)$ crystal field term and result in the configuration energy $E \sim B_{4}^{4}(c) \cos (4 \phi)$ having minima rotated by $45^{\circ}$ when changing the sign of $B_{4}^{4}(c)$ parameter. Indeed, our previously reported results show that $B_{4}^{4}(c)$ is significantly larger and of opposite sign in $\mathrm{LiYbF}_{4}$ compared to $\mathrm{LiErF}_{4}$ [13].

The powder sample of $\mathrm{LiYbF}_{4}$ was measured as a function of temperature in fine steps across the thermal phase transition. Figure 3(b) shows how the magnetic intensity of the (001) reflection decreases with temperature. As expected from ac susceptibility and heat capacity measurements, magnetic order disappears above $136 \mathrm{mK}$. Singlecrystal measurements as a function of transverse field are shown in Fig. 3(c). At $T_{\text {base }}=70 \mathrm{mK}$, a field of around $0.43 \mathrm{~T}$ suppresses the (100) magnetic peak. A small contribution from critical scattering is observed as tails of the main peak. The neutron scattering measurements of $\mathrm{LiYbF}_{4}$ reaffirm the phase diagram found from ac susceptibility in Fig. 1.

The evolution of the magnetic Bragg peak intensities with temperature and field are shown in Fig. 4(a). Continuous onset and smooth evolution of the order parameter is observed with both temperature and field.

For both the powder and single-crystal data we have considered a model consisting of (i) a Lorentzian line shape to describe the critical fluctuations close to the phase 

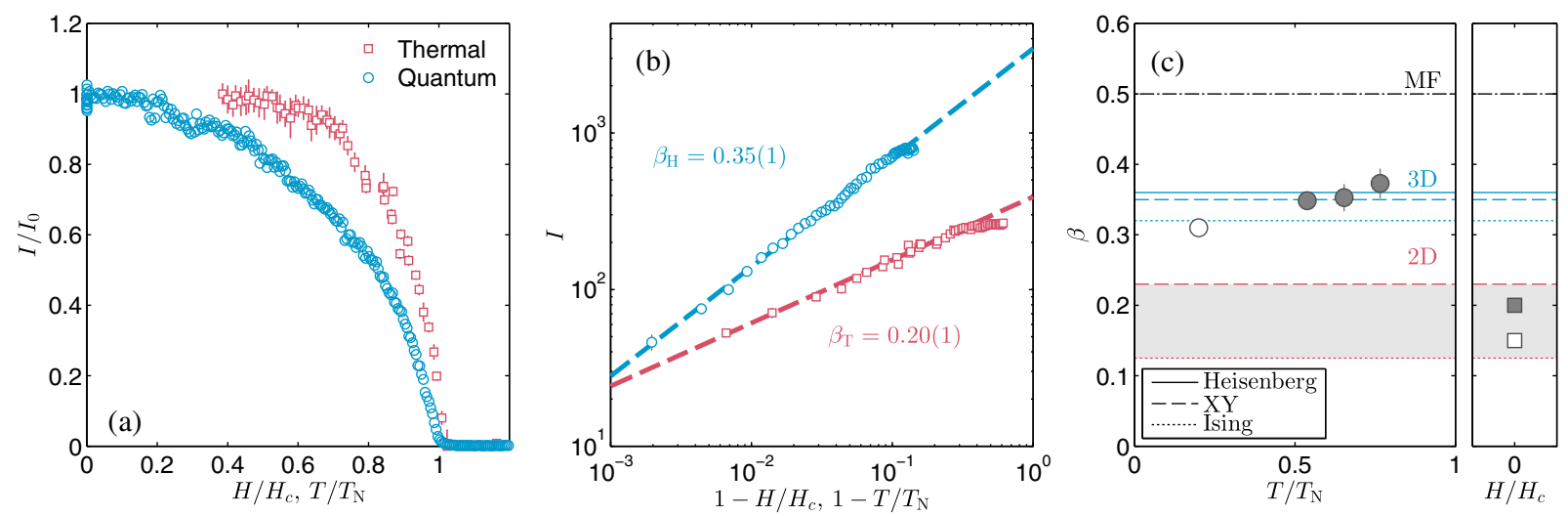

FIG. 4. (a) Evolution of the zero-field Bragg peak intensity as a function of temperature and the Bragg peak intensity as a function of the field at $50 \mathrm{mK}$. (b) Determination of the order-parameter critical exponent for the thermal classical phase transition and the quantum transition. (c) Extraction of the critical exponent $\beta_{H}$ at different temperatures is plotted by circles. The critical exponent $\beta_{T}$ is shown by squares in the panel on the right. Filled data points represent exponents found in this work for LiYbF $\mathrm{L}_{4}$, in addition empty symbols denote $\mathrm{LiErF}_{4}$ results, after Ref. [12]. Dashed lines and dotted horizontal lines correspond to critical exponents of 3D [20] and 2D [21,22] universality classes, respectively. The expected mean-field (MF) result of $\beta=0.5$ is also plotted.

transition and (ii) a delta function to account for long-range order. Both of these were then convoluted by a Gaussian, representing the instrumental resolution. The strength of scattering from critical fluctuations is rather weak and within the measured resolution and statistics cannot be refined to extract further exponents in either powder or single-crystal data. The amplitude of the convoluted delta function $\sigma$ corresponds to the square of the order parameter, i.e., staggered magnetization. Therefore, sufficiently close to the phase boundary, $\sigma \propto t^{2 \beta_{T}}$ for a zero-field measurement and $\sigma \propto h^{2 \beta_{H}}$, where $h=1-H / H_{c}$ on sweeping magnetic field at constant temperature. From such treatment we obtain the results shown in Fig. 4(b), where squares and circles are for thermal and quantum critical exponents, respectively. Fitting the data to a power law, we obtain $\beta_{T}=0.20(1)$ and $\beta_{H}=0.35(1)$.

The base temperature of $70 \mathrm{mK}$ at which the field was swept to cross the quantum phase transition may appear rather high as $T_{\text {base }} \simeq 0.5 T_{N}$. For $\mathrm{LiErF}_{4}$, on the other hand, the $\beta_{H}$ was extracted at $T_{\text {base }} \simeq 0.2 T_{N}$. To ensure that the extracted $\beta_{H}=0.35(1)$ is correct and not affected by thermal fluctuations, we followed the field evolution of the (100) Bragg peak at a few higher temperatures. We find, as shown in Fig. 4(c), no appreciable change in $\beta_{H}$ in the temperature range studied. This assertion is further corroborated by the heat capacity measurements, shown in Fig. 2, where the thermal critical region is found above around $0.8 T_{N}$. Comparing the critical exponents to tabulated results [20-22], it is clear that the quantum transition falls in the $\beta=0.32-0.36$ range predicted for 3D models. While the 2D $X Y / h_{4}$ model predicts $\beta=0.125-0.23$, bound by $2 \mathrm{D}$ Ising and $X Y$ transitions, which best describes the thermal phase transition [22].

Such dimensional reduction has been hinted at from studies of other dipolar systems. A good example is $R \mathrm{Ba}_{2} \mathrm{Cu}_{3} \mathrm{O}_{7-\delta}$ whose dipolar interactions were the focus of some theoretical work $[23,24]$. It was argued twodimensional behavior is strongly related to the spacing of basal planes with a crossover from three-dimensional behavior around $c / a>2.5$. However, relatively strong exchange coupling as well as superconductivity makes this system more complicated to separate the influence of the dipolar interaction. We hypothesize that systems such as $R \mathrm{PO}_{4}\left(\mathrm{MoO}_{3}\right)_{12} \cdot 30 \mathrm{H}_{2} \mathrm{O}$, where rare-earth ions form a diamond lattice, would also be a good candidate to examine quantum criticality due to strong dipolar and weak exchange interactions [25]. Quantum spin fluctuations of dipolar-coupled antiferromagnetism have already been suggested to play a major role in these systems [26].

To conclude, dipolar-coupled $\mathrm{LiYbF}_{4}$ undergoes a thermal transition into the bilayer, $X Y$ antiferromagnetically ordered phase, where the critical exponents follow the 2D $X Y / h_{4}$ universality class despite the lack of apparent two dimensionality in the structure. Applying a transverse magnetic field suppresses the order, inducing a quantum phase transition into a paramagnetic state, which scales according to $(2+1) \mathrm{D}$ universality. These observations are in accordance with those for $\mathrm{LiErF}_{4}$ with largely different crystal field environment, $T_{N}$, and hyperfine interactions. Our results, therefore, experimentally establish that the dimensional reduction is a universal feature of dipolarcoupled quantum antiferromagnets on the distorted diamondlike lattice and are likely to be applicable to a vast range of seemingly different systems. While it may be premature to conclude that dimensional reduction is universal to other lattices, the challenge is now to find a dipolar-coupled antiferromagnet without it.

We are grateful to B. Klemke for his technical support. We would like to thank J. O. Piatek for his help in setting up the ac susceptibility measurements and I. Živković for helpful discussions. We are indebted to J.S. White, 
M. Zolliker, and M. Bartkowiak for their assistance during preliminary measurements on the TASP spectrometer at SINQ, PSI. This work was funded by the Swiss National Science Foundation and its Sinergia network MPBH, Marie Curie Action COFUND (EPFL Fellows), and European Research Council Grant CONQUEST. This work is partially supported by Grants-in-Aid for Scientific Research (No. 25707030 and No. 15K13515) and Program for Advancing Strategic International Networks to Accelerate the Circulation of Talented Researchers (No. R2604) from the Japanese Society for the Promotion of Science. We thank HZB for the allocation of neutron radiation beam time. This project has received funding from the European Union's Seventh Framework Programme for research, technological development, and demonstration under the NMI3-II Grant No. 283883. M. M. was partly supported by Marie Sklodowska Curie Action, International Career Grant through the European Commission and Swedish Research Council (VR), Grant No. INCA-2014-6426.

*peter.babkevich@epfl.ch

[1] H. E. Stanley, Introduction to Phase Transitions and Critical Phenomena (Oxford University Press, New York, 1987), p. 336.

[2] S. Sondhi, S. Girvin, J. Carini, and D. Shahar, Rev. Mod. Phys. 69, 315 (1997).

[3] M. Vojta, Rep. Prog. Phys. 66, 2069 (2003).

[4] S. Sachdev, Quantum Phase Transitions (Wiley Online Library, 2007).

[5] E. F. Shender, Zh. Eksp. Teor. Fiz. 83, 326 (1982).

[6] C. L. Henley, Phys. Rev. Lett. 62, 2056 (1989).

[7] A. Orendáčová, M. Orendáč, and A. Feher, Czech. J. Phys. 52, 307 (2002).

[8] T. Schustereit, S. L. Müller, T. Schleid, and I. Hartenbach, Crystals 1, 244 (2011).

[9] D. Bitko, T. F. Rosenbaum, and G. Aeppli, Phys. Rev. Lett. 77, 940 (1996).

[10] H. M. Rønnow, R. Parthasarathy, J. Jensen, G. Aeppli, T. F. Rosenbaum, and D. F. McMorrow, Science 308, 389 (2005).
[11] I. Kovacevic, P. Babkevich, M. Jeong, J. O. Piatek, G. Boero, and H. M. Rønnow (to be published).

[12] C. Kraemer, N. Nikseresht, J. O. Piatek, N. Tsyrulin, B. D. Piazza, K. Kiefer, B. Klemke, T. F. Rosenbaum, G. Aeppli, C. Gannarelli, K. Prokes, A. Podlesnyak, T. Strässle, L. Keller, O. Zaharko, K. W. Krämer, and H. M. Rønnow, Science 336, 1416 (2012).

[13] P. Babkevich, A. Finco, M. Jeong, B. D. Piazza, I. Kovacevic, G. Klughertz, K. W. Krämer, C. Kraemer, D. T. Adroja, E. Goremychkin, T. Unruh, T. Strässle, A. Di Lieto, J. Jensen, and H. M. Rønnow, Phys. Rev. B 92, 144422 (2015).

[14] M. Le, D. Quintero-Castro, R. Toft-Petersen, F. Groitl, M. Skoulatos, K. Rule, and K. Habicht, Nucl. Instrum. Methods Phys. Res., Sect. A 729, 220 (2013).

[15] We note that the different techniques gave slightly different values of $T_{N}$, within $10 \mathrm{mK}$. We attribute this to differing thermometer calibrations and possibly small thermal gradients between sample and thermometer. This does not affect the extracted exponents nor the main conclusions of this Letter.

[16] See Supplemental Material at http://link.aps.org/ supplemental/10.1103/PhysRevLett.116.197202, which includes Refs. [12,13,17-19].

[17] J. Rodriguez-Carvajal, Physica (Amsterdam) 192B, 55 (1993).

[18] R. E. Thoma, G. D. Brunton, R. A. Penneman, and T. K. Keenan, Inorg. Chem. 9, 1096 (1970).

[19] J. Rodriguez-Carvajal, https://www.ill.eu/sites/fullprof.

[20] S. W. Lovesey, The Theory of Neutron Scattering from Condensed Matter (Clarendon Press, Oxford, 1986).

[21] F. Kagawa, K. Miyagawa, and K. Kanoda, Nature (London) 436, 534 (2005).

[22] A. Taroni, S. T. Bramwell, and P. C. W. Holdsworth, J. Phys. Condens. Matter 20, 275233 (2008).

[23] K. De'Bell and J. P. Whitehead, J. Phys. Condens. Matter 3, 2431 (1991).

[24] A. B. MacIsaac, J. P. Whitehead, K. De'Bell, and K. S. Narayanan, Phys. Rev. B 46, 6387 (1992).

[25] J. T. Van der Noordaa and L. R. Corruccini, J. Low Temp. Phys. 121, 81 (2000).

[26] S. J. White, M. R. Roser, J. Xu, J. T. van der Noordaa, and L. R. Corruccini, Phys. Rev. Lett. 71, 3553 (1993). 